\title{
Sinc-Chebyshev Collocation Method for a Class of Fractional Diffusion-Wave Equations
}

\author{
Zhi Mao, ${ }^{1,2}$ Aiguo Xiao, ${ }^{1}$ Zuguo Yu, ${ }^{1}$ and Long Shi ${ }^{1}$ \\ ${ }^{1}$ Hunan Key Laboratory for Computation and Simulation in Science and Engineering and Key Laboratory of Intelligent Computing \\ and Information Processing of Ministry of Education, Xiangtan University, Xiangtan, Hunan 411105, China \\ ${ }^{2}$ Mathematics and Information Engineering Department, Tongren University, Tongren, Guizhou 554300, China
}

Correspondence should be addressed to Zhi Mao; mzxtu1982@163.com

Received 9 January 2014; Accepted 17 February 2014; Published 1 April 2014

Academic Editors: C. Li, A. Sikorskii, and S. B. Yuste

Copyright (C) 2014 Zhi Mao et al. This is an open access article distributed under the Creative Commons Attribution License, which permits unrestricted use, distribution, and reproduction in any medium, provided the original work is properly cited.

\begin{abstract}
This paper is devoted to investigating the numerical solution for a class of fractional diffusion-wave equations with a variable coefficient where the fractional derivatives are described in the Caputo sense. The approach is based on the collocation technique where the shifted Chebyshev polynomials in time and the sinc functions in space are utilized, respectively. The problem is reduced to the solution of a system of linear algebraic equations. Through the numerical example, the procedure is tested and the efficiency of the proposed method is confirmed.
\end{abstract}

\section{Introduction}

Fractional models have been increasingly shown by many scientists to describe adequately the problems with memory and nonlocal properties in fluid mechanics, viscoelasticity, physics, biology, chemistry, finance, and other areas of applications [1-6]. In particular, the fractional diffusion-wave equation has been used to model many important physical phenomena ranging from amorphous, colloid, glassy, and porous materials through fractals, percolation clusters, and random and disordered media to comb structures, dielectrics and semiconductors, polymers, and biological systems [710]. It is a generalization of the classical diffusion-wave equation by replacing the integer-order time derivative with a fractional derivative of order $\alpha(1<\alpha<2)$. This equation can be derived from the anomalous superdiffusion in continuous time random walk which is generally nonMarkovian processes [11].

Although the considerable work on the numerical solution of fractional diffusion equations has been done [1215], there are very limited numerical methods for solving the fractional diffusion-wave equations [16-18]. However, all the above mentioned papers dealt with the fractional diffusion-wave equations by finite difference methods. It is well known that any algorithm based on the finite difference discretization of a fractional derivative has to take into account its memory or nonlocal structure; thus this means a high storage requirement [19].

In the present paper, we consider the following differential equation with the Caputo fractional derivative and a variable coefficient:

$$
\begin{array}{r}
\frac{\partial^{\alpha} u(x, t)}{\partial t^{\alpha}}=a(x, t) \frac{\partial^{2} u(x, t)}{\partial x^{2}}+f(x, t), \\
a<x<b, 0<t \leq \tau,
\end{array}
$$

with the initial conditions,

$$
u(x, 0)=\phi(x), \quad \frac{\partial u(x, 0)}{\partial t}=\psi(x), \quad a<x<b,
$$

and the boundary conditions,

$$
u(a, t)=0, \quad u(b, t)=0, \quad 0<t \leq \tau,
$$

where $x \in[a, b]$ and $t \in(0, \tau]$ are space and time variables, respectively, $a(x, t)$ is a continuous function, and $f(x, t)$ denotes the field variable. For $1<\alpha<2$, the 
fractional equation (1) is known as the fractional diffusionwave equation which fills the gaps between the diffusion equation and wave equation $[16,20]$.

We develop a sinc-Chebyshev collocation method to solve numerically problem (1) with (2) and (3). Since a fractional derivative is a nonlocal operator, it is natural to consider a global scheme such as the collocation method for its numerical solution $[19,21]$. The required approximate solution is expanded as a series with the elements of shifted Chebyshev polynomials in time and sinc functions in space with unknown coefficients. By utilizing the collocation technique and some properties of the shifted Chebyshev polynomials and sinc functions, the problem is reduced to the solution to a system of linear algebraic equations. And a matrix representation of the system is obtained to calculate the solution. The presented method is effective and convenient.

The remainder of this paper is organized as follows: in the next section, we introduce some necessary definitions and relevant results for developing this method. Section 3 is devoted to constructing and analyzing the numerical algorithm. As a result, a system of linear algebraic equations is formed and the solution of the considered problem is obtained. In Section 4, the numerical example is given to demonstrate the effectiveness and convergence of the proposed method. A brief conclusion is given in the final section.

\section{Notations and Some Preliminary Results}

In this section, we introduce some basic definitions and derive several preliminary results for developing the presented method.

\subsection{The Caputo Fractional Derivative}

Definition 1 (see [22]). Let $\alpha \in \mathbb{R}_{+}$. The operator $J_{a}^{\alpha}$ defined on $L_{1}[a, b]$ by

$$
J_{a}^{\alpha} f(t)=\frac{1}{\Gamma(\alpha)} \int_{a}^{t}(t-s)^{\alpha-1} f(s) d s
$$

for $a \leq t \leq b$ is called the Riemann-Liouville fractional integral operator of order $\alpha$. For, $\alpha=0$, we set $J_{a}^{0}:=I$, that is, the identity operator.

Definition 2 (see [22]). Let $\alpha \in \mathbb{R}_{+}$and $n=\lceil\alpha\rceil$. The Caputo fractional differential operator ${ }^{C} D_{a}^{\alpha}$ for $a \leq t \leq b$ is defined as

$$
\begin{aligned}
{ }^{C} D_{a}^{\alpha} f(t) & =J_{a}^{n-\alpha} D^{n} f(t) \\
& =\frac{1}{\Gamma(n-\alpha)} \int_{a}^{t}(t-s)^{n-\alpha-1} f^{(n)}(s) d s .
\end{aligned}
$$

2.2. The Composite Translated Sinc Functions. The sinc functions and their properties are discussed in $[23,24]$. For any $h>0$, the translated sinc functions with equidistant space nodes are given as

$$
S(k, h)(z)=\operatorname{sinc}\left(\frac{z-k h}{h}\right), \quad k=0, \pm 1, \pm 2, \ldots,
$$

where the sinc functions are defined on the whole real line by

$$
\operatorname{sinc}(x)= \begin{cases}\frac{\sin (\pi x)}{\pi x}, & x \neq 0 \\ 1, & x=0\end{cases}
$$

If $f$ is defined on $\mathbb{R}$, then for any $h>0$ the series

$$
C(f, h)(z)=\sum_{k=-\infty}^{\infty} f(k h) S(k, h)(z)
$$

is called the Whittaker cardinal expansion of $f$ whenever this series converges. $f$ can be approximated by truncating (8).

To construct our needed approximations on the interval $[a, b]$, we choose

$$
\varphi(x)=\ln \left(\frac{x-a}{b-x}\right)
$$

which maps the finite interval $[a, b]$ onto $\mathbb{R}$. The basic functions on $[a, b]$ are taken to be the composite translated sinc functions:

$$
S_{\varphi}(k, h)(x)=S(k, h)(\varphi(x))=\operatorname{sinc}\left(\frac{\varphi(x)-k h}{h}\right) .
$$

Thus we may define the inverse image of the equidistant space node $\{i h\}$ as

$$
x_{i}=\varphi^{-1}(i h)=\frac{a+b e^{i h}}{1+e^{i h}}, \quad i=0, \pm 1, \pm 2, \ldots
$$

The class of functions such that the known exponential convergence rate exists for the sinc interpolation is denoted by $B\left(D_{E}\right)$ and defined in the following text.

Definition 3 (see [21]). Let $B\left(D_{E}\right)$ be the class of functions $f$ which are analytic in $D_{E}$ and satisfy

$$
\int_{\varphi^{-1}(x+L)}|f(z) d z| \longrightarrow 0, \quad x \longrightarrow \pm \infty
$$

where $L=\{i v:|v|<d \leq \pi / 2\}$, and

$$
\int_{\partial D_{E}}|f(z) d z|<\infty
$$

on the boundary of $D_{E}$ (denoted $\left.\partial D_{E}\right)$.

Theorem 4 (see $[21,23])$. If $\varphi^{\prime} f \in B\left(D_{E}\right)$, then, for all $x \in$ $[a, b]$,

$$
\left|f(x)-\sum_{k=-\infty}^{\infty} f\left(x_{k}\right) S_{\varphi}(k, h)(x)\right| \leq \frac{2 N\left(\varphi^{\prime} f\right)}{\pi d} e^{-\pi d / h} .
$$


Further, one assumes that there are positive constants $C$ and $\beta$ so that $|f(x)| \leq C \exp (-\beta|\varphi(x)|)$. And if one selects $h=$ $\sqrt{\pi d / \beta N} \leq 2 \pi d / \ln 2$, then,

$$
\begin{gathered}
\left|\frac{d^{m} f(x)}{d x^{m}}-\sum_{k=-N}^{N} f\left(x_{k}\right) \frac{d^{m}}{d x^{m}} S_{\varphi}(k, h)(x)\right| \\
\leq K N^{(m+1) / 2} \exp (-\sqrt{\pi d \beta N})
\end{gathered}
$$

for all $m=0,1, \ldots, n$.

The above expressions show that the sinc interpolation on $B\left(D_{E}\right)$ converges exponentially. We also require the following derivatives of the composite translated sinc functions evaluated at the nodes. Consider

$$
\begin{gathered}
\delta_{k, i}^{(0)}=\left.\left[S_{\varphi}(k, h)(x)\right]\right|_{x=x_{i}}= \begin{cases}1, & k=i, \\
0, & k \neq i,\end{cases} \\
\delta_{k, i}^{(1)}=\left.\frac{d}{d \varphi}\left[S_{\varphi}(k, h)(x)\right]\right|_{x=x_{i}}= \begin{cases}0, & k=i, \\
\frac{(-1)^{i-k}}{(i-k) h}, & k \neq i,\end{cases} \\
\delta_{k, i}^{(2)}=\left.\frac{d^{2}}{d \varphi^{2}}\left[S_{\varphi}(k, h)(x)\right]\right|_{x=x_{i}}= \begin{cases}\frac{-\pi^{2}}{3 h^{2}}, & k=i, \\
\frac{-2(-1)^{i-k}}{(i-k)^{2} h^{2}}, & k \neq i .\end{cases}
\end{gathered}
$$

2.3. The Shifted Chebyshev Polynomials. The Chebyshev polynomials $\left\{T_{i}(\sigma) ; i=0,1, \ldots\right\}$ are a well-known family of orthogonal polynomials defined on the interval $[-1,1]$ and can be determined with the aid of the recurrence formulae $[25,26]$ :

$$
\begin{gathered}
T_{n+1}(\sigma)=2 x T_{n}(\sigma)-T_{n-1}(\sigma), \quad n=1,2, \ldots, \\
T_{0}(\sigma)=1, \quad T_{1}(\sigma)=\sigma .
\end{gathered}
$$

In order to use these polynomials on the interval $t \in$ $[0, \tau]$, it is necessary to define the so-called shifted Chebyshev polynomials by the variable substitution: $\sigma=(2 t / \tau)-1$. Let the shifted Chebyshev polynomials $T_{i}((2 t / \tau)-1)$ be denoted by $T_{\tau, i}(t)$. The analytic form of the shifted Chebyshev polynomials $T_{\tau, i}(t)$ is given by

$$
\begin{gathered}
T_{\tau, i}(t)=i \sum_{k=0}^{i}(-1)^{i-k} \frac{(i+k-1) ! 2^{2 k}}{(i-k) !(2 k) ! \tau^{k}} t^{k}, \quad i=1,2, \ldots \\
T_{\tau, 0}(t)=1
\end{gathered}
$$

Specially, $T_{\tau, i}(0)=(-1)^{i}$ and $T_{\tau, i}(\tau)=1$.

Caputo's fractional derivative of order $\alpha>0$ for the shifted Chebyshev polynomials $T_{\tau, i}(t)$ is given by

$$
\begin{gathered}
{ }^{C} D_{0}^{\alpha} T_{\tau, i}(t)=\sum_{k=\lceil\alpha\rceil}^{i} b_{i, k} t^{k-\alpha}, \quad i=\lceil\alpha\rceil,\lceil\alpha\rceil+1, \ldots, \\
{ }^{C} D_{0}^{\alpha} T_{\tau, i}(t)=0, \quad i=0,1, \ldots,\lceil\alpha\rceil-1,
\end{gathered}
$$

where

$$
b_{i, k}=i(-1)^{i-k} \frac{(i+k-1) ! 2^{2 k} k !}{(i-k) !(2 k) ! \tau^{k} \Gamma(k-\alpha+1)} .
$$

\section{The Derivation of the Sinc-Chebyshev Collocation Method}

In order to solve problem (1) with (2) and (3), first of all, we approximate $u(x, t)$ by the $2 m+1$ composite translated sinc functions and $n+1$ shifted Chebyshev polynomials as

$$
u_{m, n}(x, t)=\sum_{i=-m}^{m} \sum_{j=0}^{n} c_{i j} S_{\varphi}(i, h)(x) T_{\tau, j}(t) .
$$

It is noted that the approximate solution $u_{m, n}(x, t)$ satisfies the boundary conditions in (3) since $S_{\varphi}(i, h)(x), i=-m,-m+$ $1, \ldots, m$, tend to zeros when $x$ tends to $a$ and $b$. For discretizing (1) with (2), the lemma is given as follows.

Lemma 5. Let $1<\alpha<2$ and $x_{k}$ be spatial collocation points given in (11). Then the following relations hold:

$$
\begin{gathered}
\frac{\partial^{\alpha} u_{m, n}\left(x_{k}, t\right)}{\partial t^{\alpha}}=\sum_{j=2}^{n} \sum_{r=2}^{j} c_{k j} b_{j, r} t^{r-\alpha}, \\
\frac{\partial^{2} u_{m, n}\left(x_{k}, t\right)}{\partial x^{2}}=\sum_{i=-m}^{m} \sum_{j=0}^{n} c_{i j} q_{i, k} T_{\tau, j}(t), \\
\frac{\partial u_{m, n}\left(x_{k}, t\right)}{\partial t}=\sum_{j=1}^{n} \sum_{r=1}^{j} c_{k j} d_{j, r} t^{r-1},
\end{gathered}
$$

where $_{i, k}=\varphi^{\prime \prime}\left(x_{k}\right) \delta_{i, k}^{(1)}+\left[\varphi^{\prime}\left(x_{k}\right)\right]^{2} \delta_{i, k}^{(2)}$ and $d_{j, r}=j(-1)^{j-r}(r(j+$ $\left.r-1) ! 2^{2 r} /(j-r) !(2 r) ! \tau^{r}\right)$.

Proof. By (16), (20), and (22), it follows that

$$
\begin{aligned}
\frac{\partial^{\alpha} u_{m, n}\left(x_{k}, t\right)}{\partial t^{\alpha}} & =\sum_{i=-m}^{m} \sum_{j=0}^{n} c_{i j} S_{\varphi}(i, h)\left(x_{k}\right){ }^{C} D_{0}^{\alpha} T_{\tau, j}(t) \\
& =\sum_{i=-m}^{m} \sum_{j=2}^{n} c_{i j} \delta_{i, k}^{(0)} \sum_{r=2}^{j} b_{j, r} t^{r-\alpha} \\
& =\sum_{j=2}^{n} \sum_{r=2}^{j} c_{k j} b_{j, r} t^{r-\alpha} .
\end{aligned}
$$

Taking into account (17), we obtain

$$
\begin{aligned}
& \frac{\partial^{2} u_{m, n}\left(x_{k}, t\right)}{\partial x^{2}} \\
& \quad=\left.\sum_{i=-m}^{m} \sum_{j=0}^{n} c_{i j} \frac{d^{2}}{d x^{2}}\left[S_{\varphi}(i, h)(x)\right]\right|_{x=x_{k}} T_{\tau, j}(t)
\end{aligned}
$$



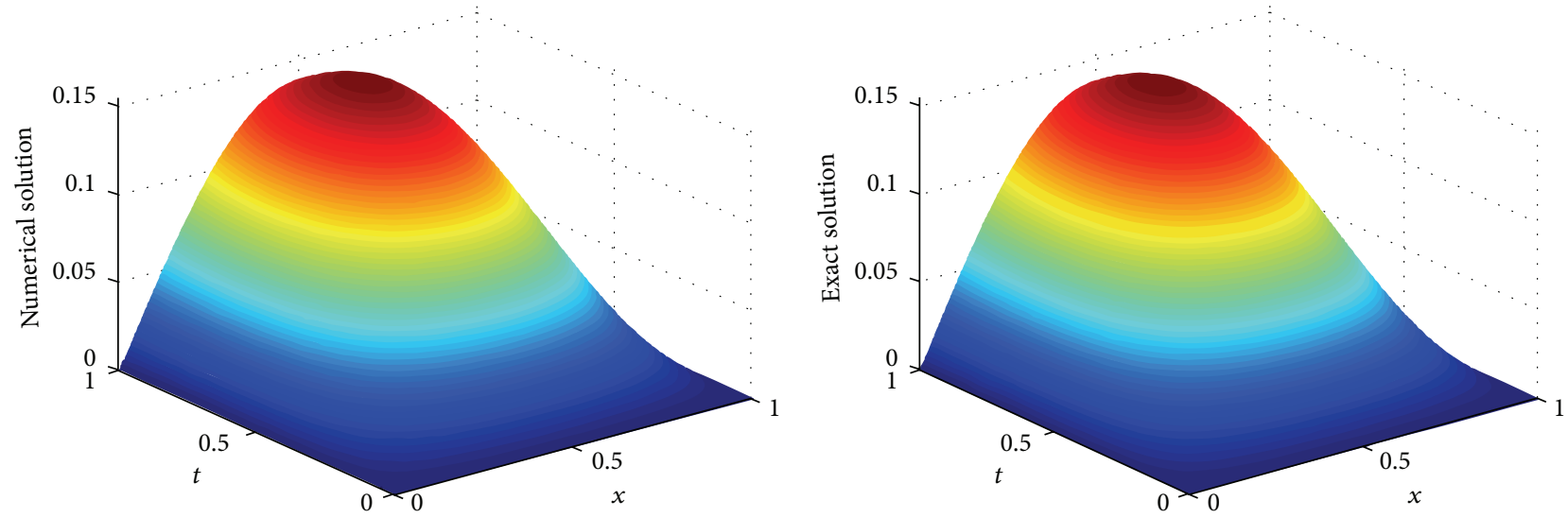

Figure 1: Comparison of the numerical and exact solution in the domain $[0,1] \times[0,1]$.

$$
\begin{aligned}
& =\sum_{i=-m}^{m} \sum_{j=0}^{n} c_{i j}\left(\varphi^{\prime \prime}\left(x_{k}\right) \delta_{i, k}^{(1)}+\left[\varphi^{\prime}\left(x_{k}\right)\right]^{2} \delta_{i, k}^{(2)}\right) T_{\tau, j}(t) \\
& =\sum_{i=-m}^{m} \sum_{j=0}^{n} c_{i j} q_{i, k} T_{\tau, j}(t) .
\end{aligned}
$$

Using (16) and (19), one has

$$
\begin{aligned}
\frac{\partial u_{m, n}\left(x_{k}, t\right)}{\partial t} & =\sum_{i=-m}^{m} \sum_{j=0}^{n} c_{i j} S_{\varphi}(i, h)\left(x_{k}\right) \frac{d}{d t} T_{\tau, j}(t) \\
& =\sum_{i=-m}^{m} \sum_{j=1}^{n} c_{i j} \delta_{i, k}^{(0)} j \sum_{r=1}^{j}(-1)^{j-r} \frac{r(j+r-1) ! 2^{2 r}}{(j-r) !(2 r) ! \tau^{r}} t^{r-1} \\
& =\sum_{j=1}^{n} \sum_{r=1}^{j} c_{k j} d_{j, r} t^{r-1} .
\end{aligned}
$$

The proof is completed.

We are now ready to solve problem (1) with (2) and (3). A collocation scheme is constructed by substituting (22) for $u(x, t)$ into (1) and evaluating the result at the points $x_{k}$ in (11) and $t_{l}$. For suitable temporal collocation points, we use the roots $t_{l}(l=1,2, \ldots, n-1)$ of the shifted Chebyshev polynomials $T_{\tau, n-1}(t)$. Therefore, using Lemma 5, we have

$$
\begin{aligned}
& \sum_{j=2}^{n} \sum_{r=2}^{j} c_{k j} b_{j, r} t_{l}^{r-\alpha} \\
& \quad-a\left(x_{k}, t_{l}\right) \sum_{i=-m j=0}^{m} \sum_{j=0}^{n} c_{i j} q_{i k} T_{\tau, j}\left(t_{l}\right)=f\left(x_{k}, t_{l}\right), \\
& k=-m,-m+1, \ldots, m, l=1,2, \ldots, n-1 .
\end{aligned}
$$

Also by applying (22) to the initial conditions (2) and collocating in $2 m+1$ points $x_{k}$, we obtain

$$
\begin{gathered}
\sum_{j=0}^{n}(-1)^{j} c_{k j}=\phi\left(x_{k}\right), \quad k=-m,-m+1, \ldots, m, \\
\sum_{j=1}^{n}(-1)^{j-1} \frac{2 j^{2}}{\tau} c_{k j}=\psi\left(x_{k}\right), \quad k=-m,-m+1, \ldots, m .
\end{gathered}
$$

To obtain a matrix representation of the above equations, we let

$$
\begin{gathered}
A=\left[\begin{array}{l}
A_{1} \\
A_{2} \\
A_{3}
\end{array}\right], \quad B=\left[\begin{array}{l}
B_{1} \\
B_{2} \\
B_{3}
\end{array}\right], \\
C=\left[c_{11}, c_{12}, \ldots, c_{1, n+1}, c_{21}, c_{22}, \ldots, c_{2, n+1}, \ldots,\right. \\
\left.c_{2 m+1,1}, c_{2 m+1,2}, \ldots, c_{2 m+1, n+1}\right]^{T}, \\
p=\left\lfloor\frac{i}{(2 m+1)}\right\rfloor, \quad s=\left\lfloor\frac{j}{(n+1)}\right\rfloor, \\
v=a\left(x_{i-p(2 m+1)-m-1}, t_{p}\right) q_{s, i-p(2 m+1)-m-1} T_{\tau, v}\left(t_{p}\right),
\end{gathered}
$$

where

$$
\begin{gathered}
A_{1}=\left(a_{i j}^{(1)}\right)_{(2 m+1) \times[(2 m+1)(n+1)]}, \\
A_{2}=\left(a_{i j}^{(2)}\right)_{(2 m+1) \times[(2 m+1)(n+1)]}, \\
A_{3}=\left(a_{i j}^{(3)}\right)_{[(2 m+1)(n-1)] \times[(2 m+1)(n+1)]}, \\
a_{i j}^{(1)}= \begin{cases}(-1)^{j-(i-1)(n+1)-1}, & 1 \leq j-(i-1)(n+1) \leq n+1, \\
0, & \text { else, }\end{cases}
\end{gathered}
$$




$$
\begin{aligned}
& a_{i j}^{(2)} \\
& =\left\{\begin{array}{r}
(-1)^{j-(i-1)(n+1)} \frac{2[j-(i-1)(n+1)-1]^{2}}{\tau}, \\
2 \leq j-(i-1)(n+1) \leq n+1, \\
0,
\end{array}\right. \\
& a_{i j}^{(3)}= \begin{cases}\Delta+\sum_{l=2}^{v} b_{v, l} t_{p}^{l-\alpha}, & s=i-p(2 m+1), v \geq 2, \\
\Delta, & \text { else, }\end{cases} \\
& B_{1}=\left[\phi\left(x_{-m}\right), \phi\left(x_{-m+1}\right), \ldots, \phi\left(x_{m}\right)\right]^{T}, \\
& B_{2}=\left[\psi\left(x_{-m}\right), \psi\left(x_{-m+1}\right), \ldots, \psi\left(x_{m}\right)\right]^{T}, \\
& B_{3}=\left[f\left(x_{-m}, t_{1}\right), f\left(x_{-m+1}, t_{1}\right), \ldots, f\left(x_{m}, t_{1}\right)\right. \text {, } \\
& f\left(x_{-m}, t_{2}\right), f\left(x_{-m+1}, t_{2}\right), \ldots, \\
& f\left(x_{m}, t_{2}\right), \ldots, f\left(x_{-m}, t_{n-1}\right), \\
& \left.f\left(x_{-m+1}, t_{n-1}\right), \ldots, f\left(x_{m}, t_{n-1}\right)\right]^{T} .
\end{aligned}
$$

So we get a system of $(2 m+1)(2 n+1)$ linear equations with $(2 m+1)(2 n+1)$ unknown parameters $c_{i j}, i=1,2, \ldots, 2 m+$ $1, j=1,2, \ldots, n+1$. And this system can be expressed in a matrix form

$$
A C=B
$$

Equation (31) can be solved easily for the unknown coefficients $c_{i j}$. Consequently $u_{m, n}(x, t)$ given in (22) can be calculated.

\section{Numerical Examples}

To validate the effectiveness of the proposed method for problem (1) with (2) and (3), we consider the example given in [16].

$$
\begin{gathered}
\frac{\partial^{\alpha} u(x, t)}{\partial t^{\alpha}}=\frac{\partial^{2} u(x, t)}{\partial x^{2}}+\sin (\pi x), \quad 0<x<1,0<t \leq 1, \\
u(x, 0)=0, \quad \frac{\partial u(x, 0)}{\partial t}=0, \quad 0<x<1, \\
u(0, t)=0, \quad u(1, t)=0, \quad 0<t \leq 1 .
\end{gathered}
$$

\begin{tabular}{|c|c|c|c|c|c|}
\hline$m \backslash(x, t)$ & $(0.1,1)$ & $(0.2,1)$ & $(0.3,1)$ & $(0.4,1)$ & $(0.5,1)$ \\
\hline 8 & 0.042229 & 0.080693 & 0.111213 & 0.130835 & 0.137592 \\
\hline 12 & 0.042615 & 0.081166 & 0.111755 & 0.131400 & 0.138168 \\
\hline 16 & 0.042720 & 0.081293 & 0.111903 & 0.131557 & 0.138330 \\
\hline 20 & 0.042752 & 0.081332 & 0.111948 & 0.131606 & 0.138379 \\
\hline Exact solution & 0.042779 & 0.081371 & 0.111997 & 0.131661 & 0.138436 \\
\hline
\end{tabular}

The exact solution of the above problem is [27]

$$
u(x, t)=\frac{1}{\pi^{2}}\left[1-E_{\alpha}\left(-\pi^{2} t^{\alpha}\right)\right] \sin (\pi x),
$$

where $E_{\alpha}(z)=\sum_{k=0}^{\infty} z^{k} / \Gamma(\alpha k+1)$ is the one-parameter Mittag-Leffler function.

To solve the above problem with $\alpha=1.7$ by using the method described in Section 3, we choose $\beta=1$ and $d=\pi / 2$, and this leads to $h=\pi / \sqrt{2 m}$. We will report the accuracy
TABLE 1: Some numerical and exact solutions at $t=1$.

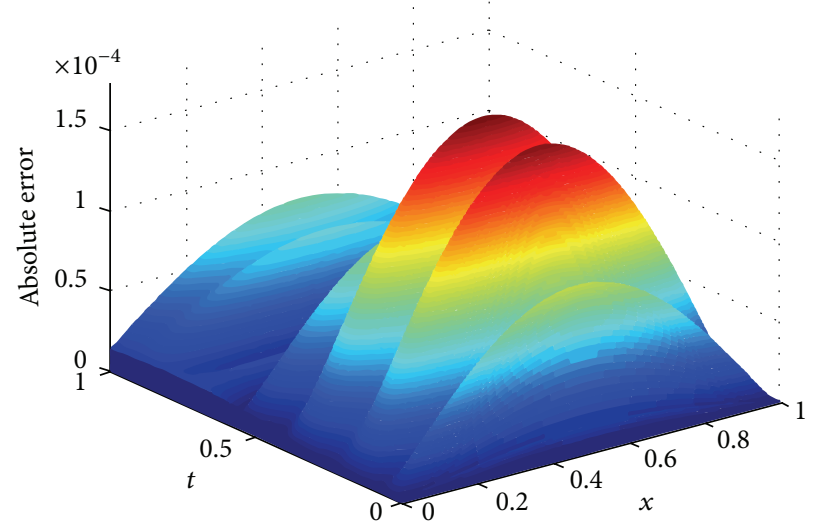

Figure 2: Plot of the absolute error.

and efficiency of the method based on the $L^{2}$-errors and $L^{\infty}$ errors. Figure 1 gives the 3D diagrams of the numerical and exact solutions on the whole computational domain $[0,1] \times$ $[0,1]$ with $m=15, n=8$. A good agreement of the numerical solution with the exact one is achieved. In Table 1, we list the numerical and exact solutions at some points for different numbers of collocation points with $n=m$. Furthermore, Figure 2 shows the absolute error function $\left|u(x, t)-u_{m, n}(x, t)\right|$ obtained by the presented method with $m=15$ and $n=8$. In Figure 3, we plot the curves of the absolute errors at $t=1$ for different numbers of collocation points. From Figures 2 and 3 , we see that the proposed method can provide accurate results only using a small number of collocation points.

To explore the dependence of errors on the parameters $m, n$, we represent the $L^{\infty}$-error and $L^{2}$-error in semi-log scale. Firstly, the computational investigation is concerned with the spatial error. To this end, we fix the polynomial degree $n=20$, a value large enough such that the error stemming from the temporal approximation is negligible. In Figure 4, we plot the error as functions of $m$, where a logarithmic scale is used for the spatial-error-axis. As expected, the error shows an exponential decay, since in this semi-log representation one observes that the error variations are approximately linear versus $m$ [19].

Now we check the temporal error, which is more interesting because of the fractional derivative in time. For a similar reason mentioned above, we fix a large enough value $m=20$ to avoid contamination of the spatial error. We present the error as a function of the shifted Chebyshev polynomial degree $n$ in Figure 5, where a logarithmic scale is now used for the temporal-error-axis. From Figure 5, it 


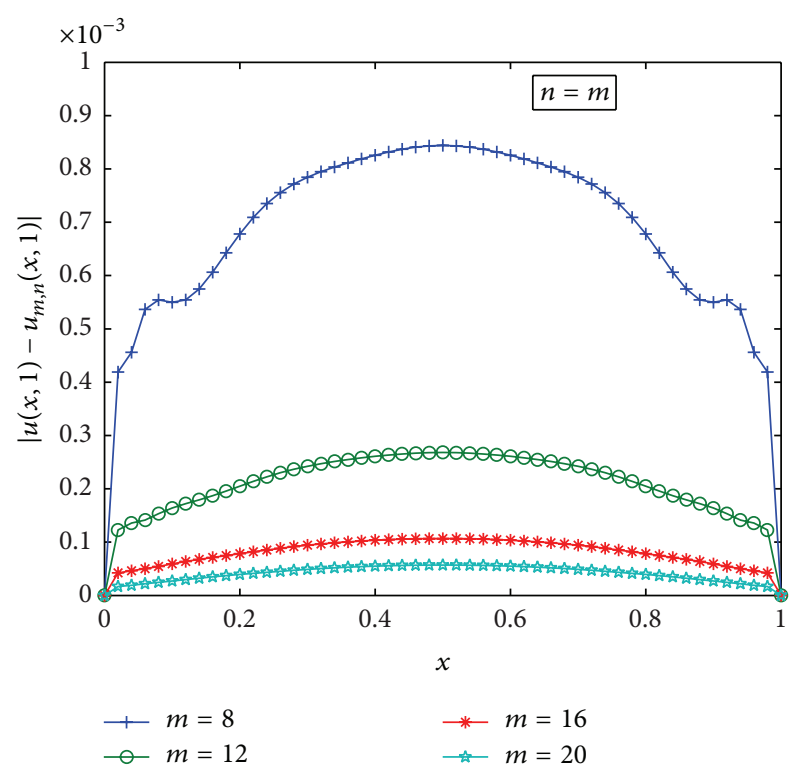

Figure 3: The error curves at $t=1$.

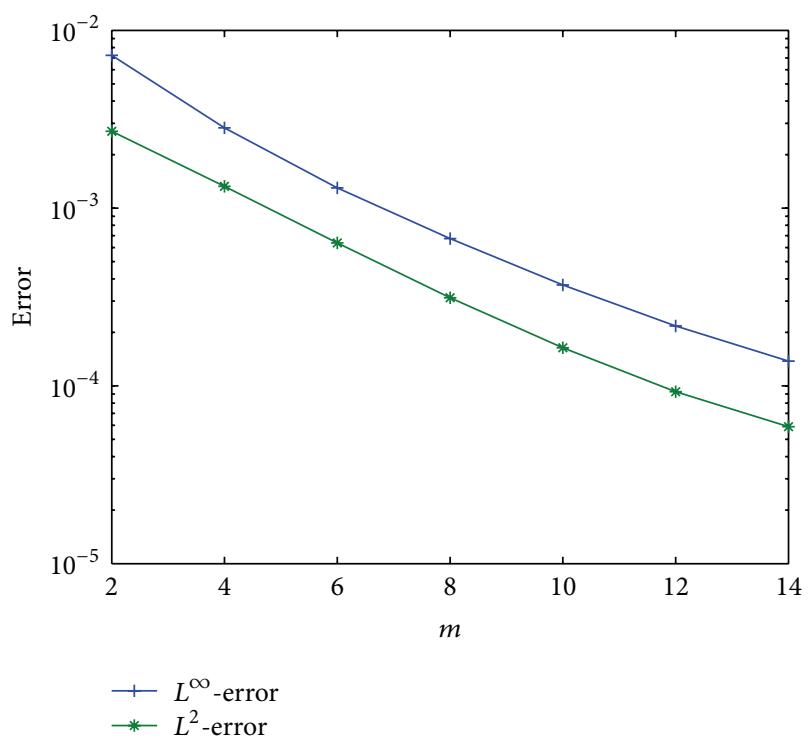

Figure 4: $L^{2}$ - and $L^{\infty}$-errors versus $m$.

is clearly observed that the temporal error depends on the discretization parameters $n$.

\section{Conclusion}

In this paper, we develop and analyze the efficient numerical methods for the fractional diffusion-wave equation. Based on the collocation technique, the sinc functions and shifted Chebyshev polynomials are used to reduce the problem to the solution of a system of linear algebraic equations. And a matrix representation of the above equations is obtained. In the numerical example, the solution obtained by this method is in excellent agreement with the exact one. The effectiveness and convergence of the presented method are confirmed

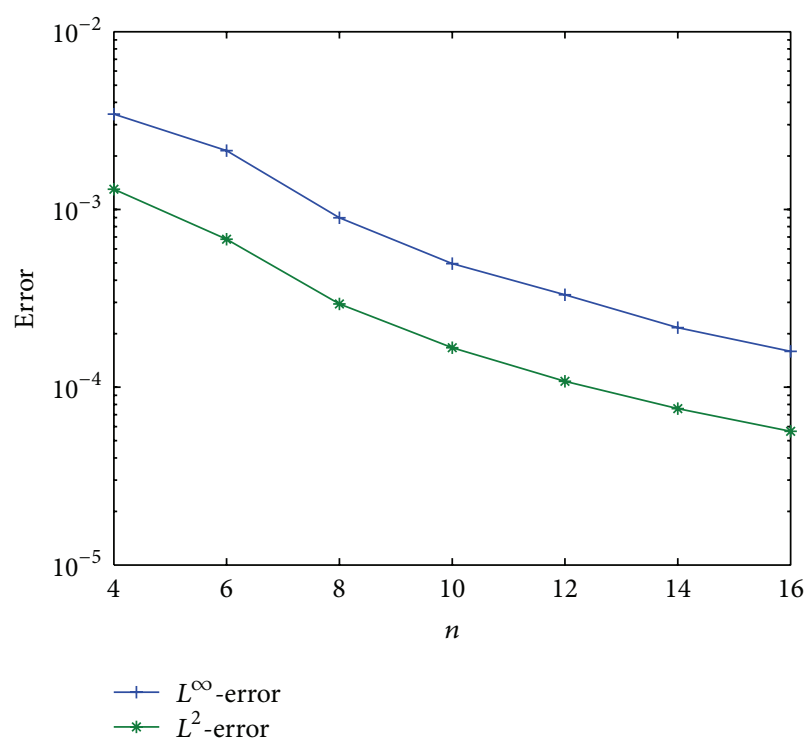

FIGURE 5: $L^{2}$ - and $L^{\infty}$-errors versus $n$.

through the numerical experimentation. One issue of future work is to develop the theory analysis of the method for the proposed fractional differential equation.

\section{Conflict of Interests}

The authors declare that there is no conflict of interests regarding the publication of this paper.

\section{Acknowledgments}

This research is supported by the National Natural Science Foundation of China (Grant nos. 11271311 and 11371016), the Chinese Program for Changjiang Scholars and Innovative Research Team in University (PCSIRT) (Grant no. IRT1179), and Hunan Province Innovation Foundation for Postgraduate (Grant no. CX2013B252).

\section{References}

[1] I. Podlubny, Fractional Differential Equations, vol. 198 of Mathematics in Science and Engineering, Academic Press, San Diego, Calif, USA, 1999.

[2] G. M. Zaslavsky, "Chaos, fractional kinetics, and anomalous transport," Physics Reports, vol. 371, no. 6, pp. 461-580, 2002.

[3] R. Metzler and J. Klafter, "The random walk's guide to anomalous diffusion: a fractional dynamics approach," Physics Reports, vol. 339, no. 1, p. 77, 2000.

[4] H. Yang, G. Luo, P. Karnchanaphanurach et al., "Protein conformational dynamics probed by single-molecule electron transfer," Science, vol. 302, no. 5643, pp. 262-266, 2003.

[5] F. Liu and K. Burrage, "Novel techniques in parameter estimation for fractional dynamical models arising from biological systems," Computers \& Mathematics with Applications, vol. 62, no. 3, pp. 822-833, 2011. 
[6] R. Magin, M. D. Ortigueira, I. Podlubny, and J. Trujillo, "On the fractional signals and systems," Signal Processing, vol. 91, no. 3, pp. 350-371, 2011.

[7] R. Metzler and J. Klafter, "The restaurant at the end of the random walk: recent developments in the description of anomalous transport by fractional dynamics," Journal of Physics A: Mathematical and General, vol. 37, no. 31, pp. R161-R208, 2004.

[8] F. Mainardi, "Fractional relaxation-oscillation and fractional diffusion-wave phenomena," Chaos, Solitons \& Fractals, vol. 7, no. 9, pp. 1461-1477, 1996.

[9] J. Q. Murillo and S. B. Yuste, "An explicit difference method for solving fractional diffusion and diffusion-wave equations in the caputo form," Journal of Computational and Nonlinear Dynamics, vol. 6, no. 2, Article ID 021014, 2011.

[10] R. L. Magin, Fractional Calculus in Bioengineering, Begell House, West Redding, Conn, USA, 2006.

[11] R. Gorenflo, F. Mainardi, D. Moretti, and P. Paradisi, "Time fractional diffusion: a discrete random walk approach," Nonlinear Dynamics, vol. 29, no. 1-4, pp. 129-143, 2002.

[12] F. Liu, S. Shen, V. Anh, and I. Turner, "Analysis of a discrete nonMarkovian random walk approximation for the time fractional diffusion equation," ANZIAM Journal, vol. 46, no. 5, pp. C488C504, 2004.

[13] S. B. Yuste and L. Acedo, "An explicit finite difference method and a new von Neumann-type stability analysis for fractional diffusion equations," SIAM Journal on Numerical Analysis, vol. 42, no. 5, pp. 1862-1874, 2005.

[14] P. Zhuang, F. Liu, V. Anh, and I. Turner, "New solution and analytical techniques of the implicit numerical method for the anomalous subdiffusion equation," SIAM Journal on Numerical Analysis, vol. 46, no. 2, pp. 1079-1095, 2008.

[15] W. Chen, L. Ye, and H. Sun, "Fractional diffusion equations by the Kansa method," Computers \& Mathematics with Applications, vol. 59, no. 5, pp. 1614-1620, 2010.

[16] Z.-Z. Sun and X. Wu, "A fully discrete difference scheme for a diffusion-wave system," Applied Numerical Mathematics, vol. 56, no. 2, pp. 193-209, 2006.

[17] J. Huang, Y. Tang, L. Vázquez, and J. Yang, "Two finite difference schemes for time fractional diffusion-wave equation," Numerical Algorithms, vol. 64, no. 4, pp. 707-720, 2013.

[18] H. Ding and C. Li, "Numerical algorithms for the fractional diffusion-wave equation with reaction term," Abstract and Applied Analysis, vol. 2013, Article ID 493406, 15 pages, 2013.

[19] X. Li and C. Xu, "A space-time spectral method for the time fractional diffusion equation," SIAM Journal on Numerical Analysis, vol. 47, no. 3, pp. 2108-2131, 2009.

[20] O. P. Agrawal, "Solution for a fractional diffusion-wave equation defined in a bounded domain," Nonlinear Dynamics, vol. 29, no. 1-4, pp. 145-155, 2002.

[21] A. Saadatmandi, M. Dehghan, and M.-R. Azizi, "The sincLegendre collocation method for a class of fractional convection-diffusion equations with variable coefficients," Communications in Nonlinear Science and Numerical Simulation, vol. 17, no. 11, pp. 4125-4136, 2012.

[22] M. Weilbeer, Efficient numerical methods for fractional differential equations and their analytical background [Ph.D. thesis], Technische Universität Braunschweig, Braunschweig, Germany, 2005.

[23] J. Lund and K. L. Bowers, Sinc Methods for Quadrature and Differential Equations, SIAM, Philadelphia, Pa, USA, 1992.
[24] F. Stenger, Handbook of Sinc Numerical Methods, Chapman \& Hall/CRC Numerical Analysis and Scientific Computing, CRC Press, Boca Raton, Fla, USA, 2011.

[25] J. Shen and T. Tang, Spectral and High-Order Methods with Applications, vol. 3 of Mathematics Monograph Series, Science Press Beijing, Beijing, China, 2006.

[26] E. H. Doha, A. H. Bhrawy, and S. S. Ezz-Eldien, "Efficient Chebyshev spectral methods for solving multi-term fractional orders differential equations," Applied Mathematical Modelling, vol. 35, no. 12, pp. 5662-5672, 2011.

[27] O. P. Agrawal, "Response of a diffusion-wave system subjected to deterministic and stochastic fields," Zeitschrift für Angewandte Mathematik und Mechanik, vol. 83, no. 4, pp. 265-274, 2003. 


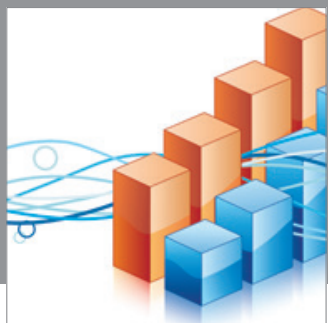

Advances in

Operations Research

mansans

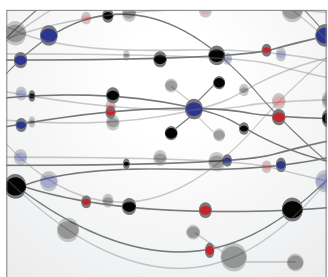

The Scientific World Journal
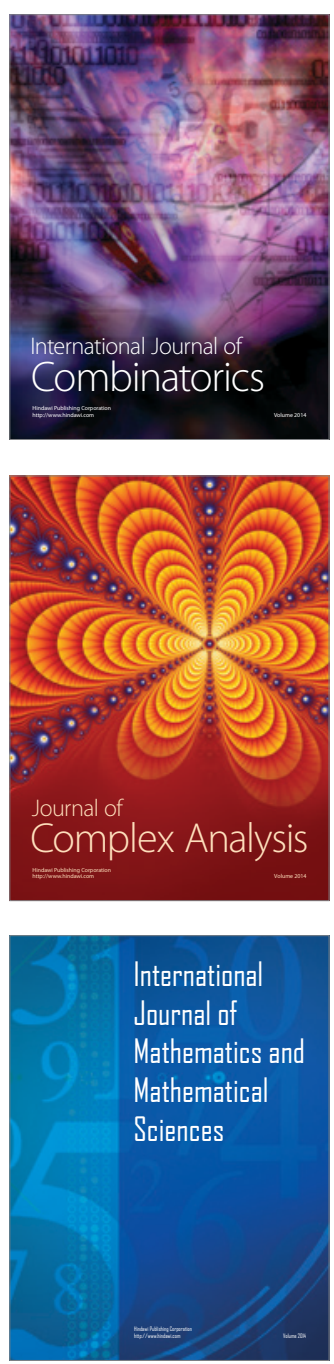
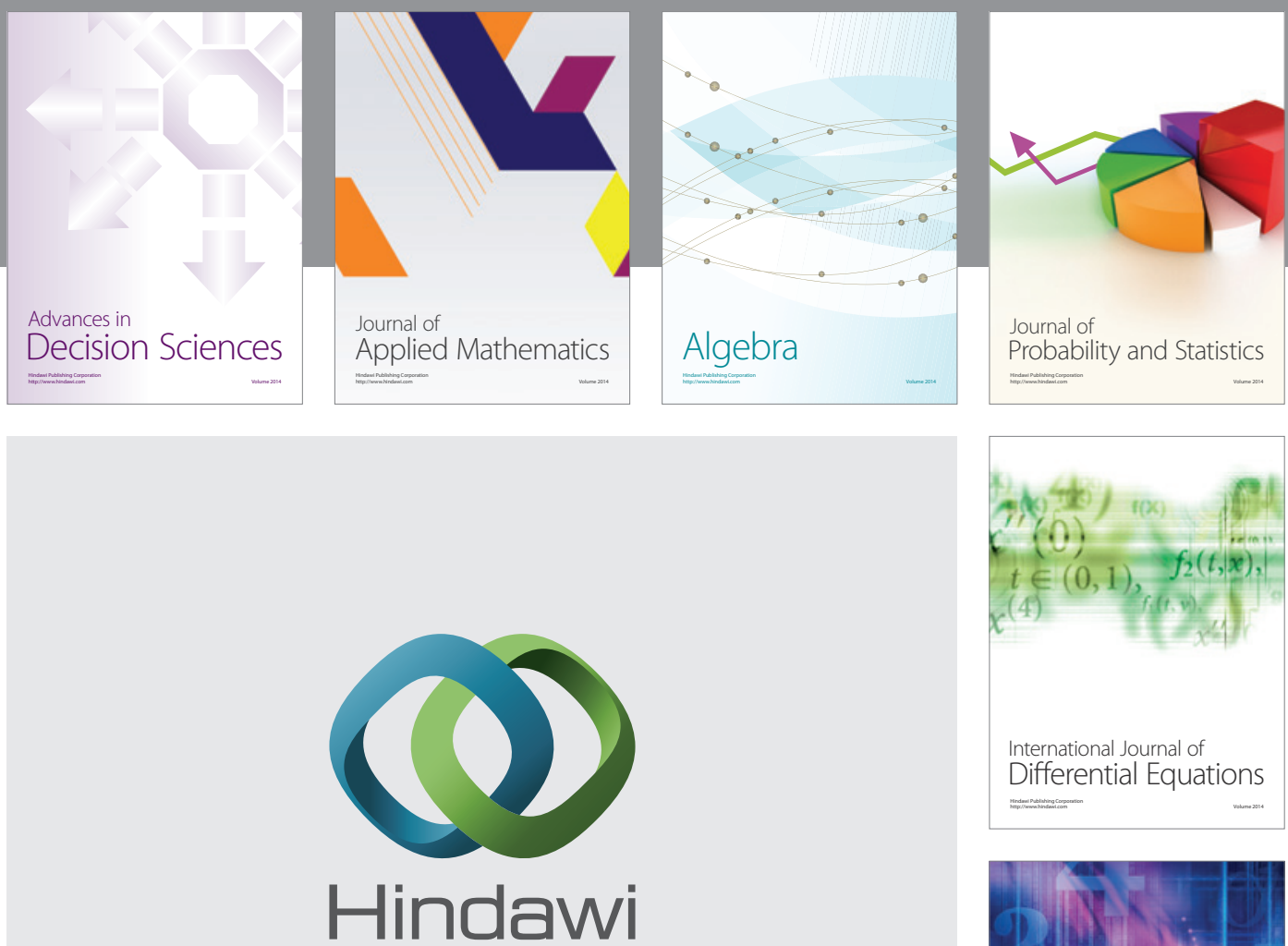

Submit your manuscripts at http://www.hindawi.com
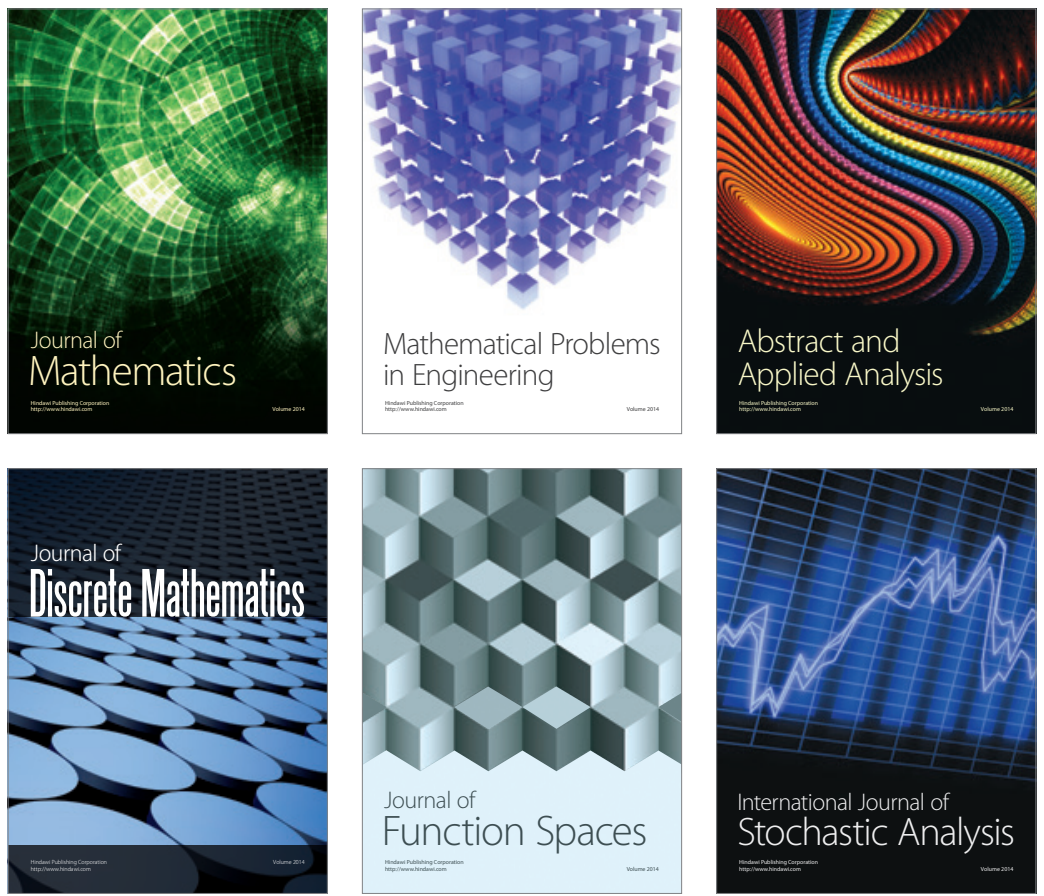

Journal of

Function Spaces

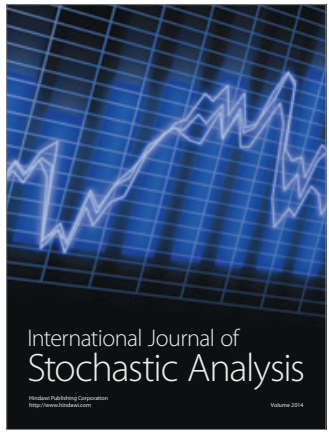

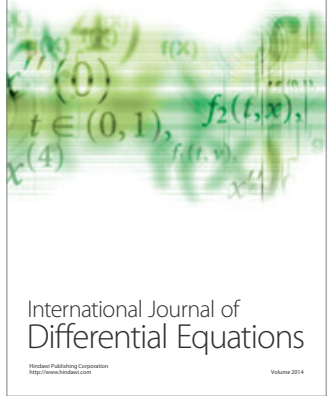
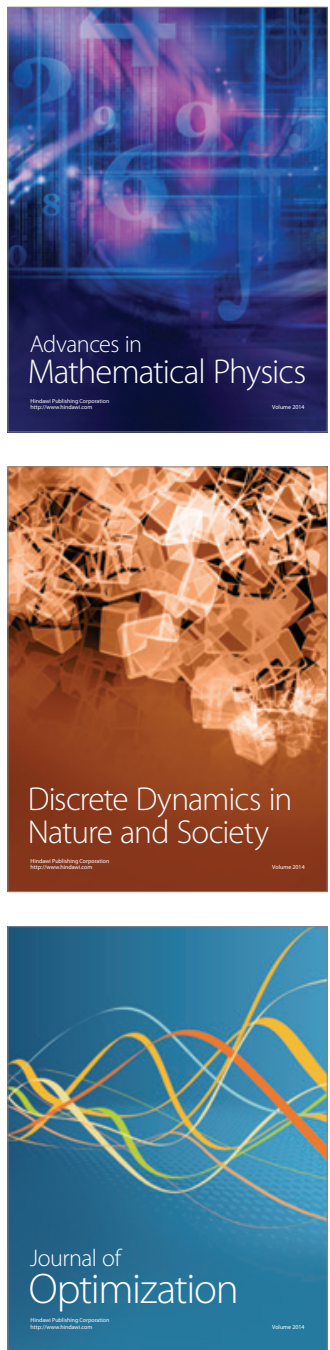